\title{
Patterning of Suitable Structures for the Investigation of the Josephson Effect in $\mathrm{YBa}_{2} \mathrm{Cu}_{3} \mathrm{O}_{7} / \mathrm{PrBa}_{2} \mathrm{Cu}_{3} \mathrm{O}_{7}$ Superlattices
}

\author{
A. Schattke, Ch. Schwan, H. Meffert, G. Jakob and H. Adrian
}

Johannes Gutenberg-Universität Mainz, Institut für Physik, Staudinger Weg 7, 55099 Mainz, Germany

\begin{abstract}
The aim of our work is to investigate the Josephson effect in $\mathrm{YBa}_{2} \mathrm{Cu}_{3} \mathrm{O}_{7-\delta} / \mathrm{PrBa}_{2} \mathrm{Cu}_{3} \mathrm{O}_{7-\delta}$ superlattices. In the case of current flow along $\mathrm{c}$ axis direction the superlattice forms an array of artificial Josephson junctions where the $\mathrm{YBa}_{2} \mathrm{Cu}_{3} \mathrm{O}_{7-\delta}$ layers are the superconducting electrodes which are separated but Josephson coupled by the $\mathrm{PrBa}_{2} \mathrm{Cu}_{3} \mathrm{O}_{7-\delta}$ sheets. In this paper we report on the preparation, characterisation and patterning of the superlattices into suitable structures via standard photolithograpy and lift-off technique.
\end{abstract}

\section{INTRODUCTION}

The existence of the intrinsic Josephson effect in $\mathrm{Bi}_{2} \mathrm{Sr}_{2} \mathrm{CaCu}_{2} \mathrm{O}_{8+\delta}$ as pointed out by Müller et al. is now well established [1,2]. Other layered compounds as $\mathrm{Tl}_{2} \mathrm{Ba}_{2} \mathrm{Ca}_{2} \mathrm{Cu}_{3} \mathrm{O}_{10+\delta}, \mathrm{Tl}_{2} \mathrm{Ba}_{2} \mathrm{CaCu}_{2} \mathrm{O}_{8+\delta}$, $(\mathrm{Bi}, \mathrm{Pb})_{2} \mathrm{Sr}_{2} \mathrm{CaCu}_{2} \mathrm{O}_{y}$ etc. show similar formation of intrinsic stacks [3,4]. In $\mathrm{Bi}_{2} \mathrm{Sr}_{2} \mathrm{CaCu}_{2} \mathrm{O}_{8+\delta}$ the electrodes of the Josephson junctions are formed by the superconducting $\mathrm{CuO}_{2}$ bilayers which are separated by the insulating $\mathrm{BiO}$ and $\mathrm{SrO}$ layers which act as the barrier. The high anisotropy of these materials is caused by the concentration of the Cooper pairs in the $\mathrm{CuO}_{2}$ planes in comparison to the insulating spacing and makes $\mathrm{Bi}_{2} \mathrm{Sr}_{2} \mathrm{CaCu}_{2} \mathrm{O}_{8+\delta}$ a good candidate for a Josephson coupled superconductor.

This relation of crystallographic to superconducting length scales implies the Josephson behaviour of this compond. An analogy can be drawn to the superlattices of $\mathrm{YBa}_{2} \mathrm{Cu}_{3} \mathrm{O}_{7-\delta} / \mathrm{PrBa}_{2} \mathrm{Cu}_{3} \mathrm{O}_{7-\delta}$. They exhibit additionally to the intrinsic modulation of the order parameter an extrinsic modulation caused by the insulating, semi- or normalconducting barrier layer. The coupling of adjacent $\mathrm{CuO}_{2}$ layers depends strongly on the barrier thickness. It is now well established that a crossover in dimensionality of the system from the coupled (3D) to the decoupled limit (2D) is achieved by increasing the barrier thickness [5]. Therefore a superlattice of the modulation 1:3 (1 unit cell $\mathrm{YBa}_{2} \mathrm{Cu}_{3} \mathrm{O}_{7-\delta}: 3$ unit cells $\mathrm{PrBa}_{2} \mathrm{Cu}_{3} \mathrm{O}_{7-\delta}$ ) shows equivalent superconducting transport properties like the anisotropic $\mathrm{Bi}_{2} \mathrm{Sr}_{2} \mathrm{CaCu}_{2} \mathrm{O}_{8+\delta}$. Increasing the $\mathrm{YBa}_{2} \mathrm{Cu}_{3} \mathrm{O}_{7-\delta}$ thickness or decreasing the barrier thickness leads to a smooth transition to $\mathrm{YBa}_{2} \mathrm{Cu}_{3} \mathrm{O}_{7-\delta}$ like behaviour. This makes superlattices good candidates for studying the Josephson effect in dependence on the barrier material. This can be controlled by doping the Praseodymium with Yttrium or the Copper with Gallium resulting in a lower or higher resistivity. Furthermore, the character of the junction will change from SNS to SS'S or SIS. Another possibility is to change the modulation of the superlattices yielding a variation of the anisotropy parameter as pointed out before. 


\section{PREPARATION AND CHARACTERISATION}

To investigate the Josephson effect in $\mathrm{YBa}_{2} \mathrm{Cu}_{3} \mathrm{O}_{7-\delta} / \mathrm{PrBa}_{2} \mathrm{Cu}_{3} \mathrm{O}_{7-\delta}$ superlattices a series of superlattices with different modulation in the range of $2: 3$ to $8: 3$ has been prepared in situ on $\mathrm{SrTiO}_{3}\left(\begin{array}{lll}1 & 0 & 0\end{array}\right)$ and $\mathrm{MgO}\left(\begin{array}{lll}1 & 0 & 0\end{array}\right)$ substrates by dc sputtering. The thicknesses range between $200 \mathrm{~nm}$ and $400 \mathrm{~nm}$. Details of the preparation and characterisation are described elsewhere [6]. After deposition the superlattices were covered with a protection layer of $400 \mathrm{~nm}$ gold. This step enables a low contact resistance and the surface is prevented from degradation during the following characterisation and etching processes. The high cristalline quality of the samples was confirmed by x-ray diffraction. The superlattice structure appears in the additional satellite lines of the diffraction pattern. Figure 1 shows the $\mathrm{x}$-ray diffraction pattern of a 18:2 superlattice taken in Bragg-Brentano-geometry. Only the part of the $\left(\begin{array}{lll}0 & 0 & 1\end{array}\right)$ and $\left(\begin{array}{lll}0 & 0 & 2\end{array}\right)$ peak is depicted. The satellites are detected up to the fourth order. The position, intensity and sharpness of these satellites allow to estimate the degree of cristalline defects and gives a high crystalline quality of the samples.

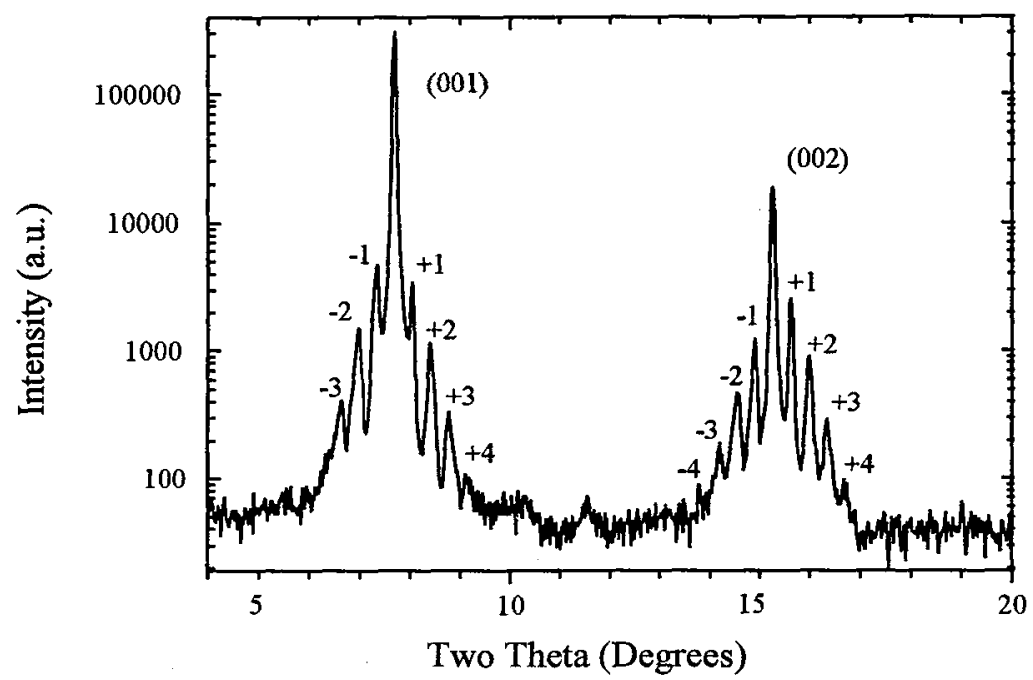

Figure 1: $X$-ray diffraction pattern of a 18:2 superlattice. The satellites, indicated with $+1,-1$ etc., appears additional to the $(00 \ell)$ reflexes due to the superlattice structure.

\section{PATTERNING}

Thin film technology offers the possibility of including the mesa device, like sub-millimeter sources or detectors, into integrated circuits. Therefore one has to design the contact leads in an in-plane geometry where the mesa has to be embedded into an insulator to separate the electrodes from each other. The patterning of the films into a suitable structure with the direction of the current along the c axis was accomplished by standard UV photolithographic technique. Figures 2a-e schematically depict subsequent steps of patterning the mesa structures. At first, before step (a) the superlattice was ion beam etched down to the substrate to define the bottom electrode. (a) In a lithographic step the area of the stack was defined ranging from $20 \times 20 \mu \mathrm{m}^{2}$ down to $2 \times 2 \mu \mathrm{m}^{2}$. (b) This mask was used as an etching mask for thinning the superlattice about $100 \mathrm{~nm}$ to $150 \mathrm{~nm}$, depending on the desired stack height, with an ion beam etching process. The beam voltage and current were $600 \mathrm{~V}$ and $0.4 \mathrm{~A} / \mathrm{cm}^{2}$ respectively. (c) The whole sample was embedded with an amorphous insulator. Usually we use $\mathrm{Mg}-\mathrm{O}$, Ce-O and amorphous $\mathrm{Y}-\mathrm{Ba}-\mathrm{Cu}-\mathrm{O}$. (d) The resist on top of the mesa is stripped 
a

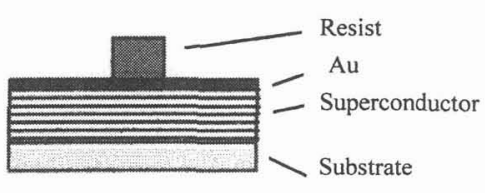

C

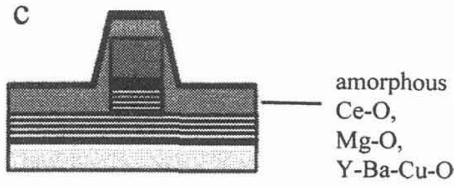

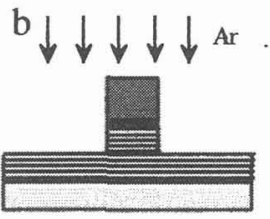

d

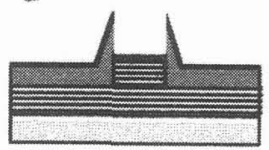

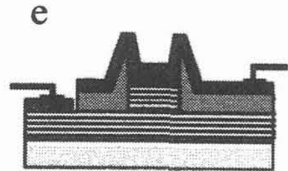

Figure 2: Fabrication process for making mesa structures. For details see text.

by a lift-off step in acetone with ultrasound and in a barrel reaktor. (e) In a last step a second gold layer is sputtered onto the sample and the voltage and current leads are patterned. It was not possible to separate the voltage and current contacts on top of the mesa and therefore no true four point measurement was achieved. Stacked series arrays of Josephson junctions were realized by this fabrication process. The most important steps of patterning the mesa structures are documented by SEM pictures. Figure 3a depicts a $20 \times 20 \mu \mathrm{m}^{2}$ mesa embedded in amorphous Y-Ba-Cu-O after a successful lift-off. The insulator is not completely removed at the edges of the structure. This effect is due to some kind of redeposition during the etching process. To reduce this effect we used an undercut resist mask which was performed in a two layer resist system (maP-275). Before the second resist layer was spinned the first layer was exposed without any mask. This yields a weakening of the lower layer resulting in very sharp undercut edges. Usually it is not trivial to observe the undercut
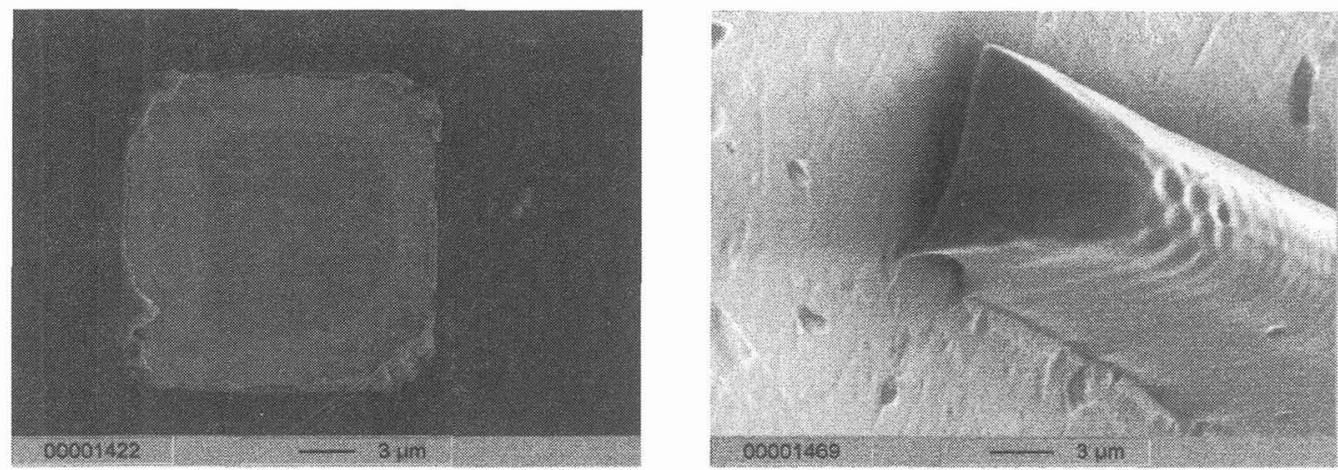

Figure 3: (a) SEM micrograph of a $20 \times 20 \mu \mathrm{m}^{2}$ mesa covered with a thin gold film after a successful lift-off. (b) Photograph of an undercut resist structure to reduce the redeposition effect during ion beam etching. For clarity the structure is not totally undercut giving a better contrast. 
of the resist from simple SEM images. In order to check it we show in fig. $3 \mathrm{~b}$ a $60^{\circ}$ tilted picture of a $20 \times 100 \mu \mathrm{m}^{2}$ mesa. Contrarely to the left side of the structure, gold sputtered over the substrate in the lower side forced the photo resist not to form an undercut. That gives a reference to the real position of the substrate with respect to the structure.

\section{CONCLUSION AND OUTLINE}

We demonstrate the successful patterning of thin film superlattices into mesa structures with dimensions in the range of $10 \mu \mathrm{m}$. To get an in-plane geometry of the contact leads the mesa is embedded in an insulator which is removed at the top of the mesa via lift-off technique. To achieve a better lift-off behaviour of the resist we perform a two layer resist mask with an undercut structure. This state of the art technology is a prerequisite to investigate the Josephson effect on superlattices with different modulation dependent on the anisotropy of the system.

\section{Acknowledgments}

Financial support by the BMBF under contract No. 13 N6437 is greatfully acknowledged.

\section{References}

[1] Kleiner, R. and Müller, P. Phys. Rev. B, 49, (1994), 1327

[2] Kleiner, R., Steinmeyer, F., Kunkel, G. and Müller, P. Phys. Rev. Lett., 68, (1992), 2394

[3] Schmidl, F., Pfuch, A., Schneidewind, H., Heinz, E., Dörrer, L., Matthes, A., Seidel, P., Hübner, U., Veith, M. and Steinbeiß, E. Supercond. Sci. Technol., 8, (1995), 740

[4] Régi, F.X., Schneck, J., Palmier, J.F. and Savary, H. J. Appl. Phys., 76, (1994), 4426

[5] Jakob, G., Przyslupski, P., Stölzel, C., Tomé-Rosa, C., Walkenhorst, A., Schmitt, M. and Adrian, H., Physica C 185-189, (1991), 2087

[6] Jakob, G., Przyslupski, P., Stölzel, C., Tomé-Rosa, C., Walkenhorst, A., Schmitt, M. and Adrian, H., Appl. Phys. Lett. C 59, (1991), 1626 